\title{
Study of Various Designs of Solid State Laser Oscillator
}

\author{
Kireet Semwal, S. C. Bhatt \\ Applied Science Department, G.B. Pant Engineering College, Pauri (Garhwal), Uttarakhand. \\ Department of Physics, HNB Garhwal Centrel University, Srinagar (Garhwal), Uttarakhand.
}

\begin{abstract}
This study concerns with the laser oscillator, threshold conditions, circulating power, and the Gaussian beam profile. Laser oscillator is a crucial device, which provides the feedback necessary to make a laser work. The regenerative laser oscillator is essentially a combination of two basic components: an optical amplifier, and an optical resonator, which serves to provide highly selective feedback. The optical resonator, comprised of two opposing plane-parallel or curved mirrors at right angles to the axis of active material, performs function of the feedback element, by coupling back in phase a portion of the signal emerging from the amplifing medium. The photons are reflected back and forth for many passes through the rod (amplifying medium), stimulating more and more emission on each pass. The mirrors can be gently curved so they tend to keep the light concentration inside the rod. One of the mirrors is $100 \%$ reflective, but the other mirror transmits part of the light hitting it. This transmitted light is the output beam from the laser
\end{abstract}

Keywords: - Laser oscillator, optical feedback, Gaussian Beam, Laser Modes, TEM.

\section{INTRODUCTION}

Laser oscillator is a crucial device, which provides the feedback necessary to make a laser work. The regenerative laser oscillator is essentially a combination of two basic components: an optical amplifier, and an optical resonator, which serves to provide highly selective feedback. The optical resonator, comprised of two opposing plane-parallel or curved mirrors at right angles to the axis of active material shown in Figure-1, performs function of the feedback element, by coupling back in phase a portion of the signal emerging from the amplifying medium. The photons are reflected back and forth for many passes through the rod (amplifing medium), stimulating more and more emission on each pass. The mirrors can be gently curved so they tend to keep the light concentration inside the rod. One of the mirrors is $100 \%$ reflective, but the other mirror transmits part of the light hitting it. This transmitted light is the output beam from the laser [1][2].
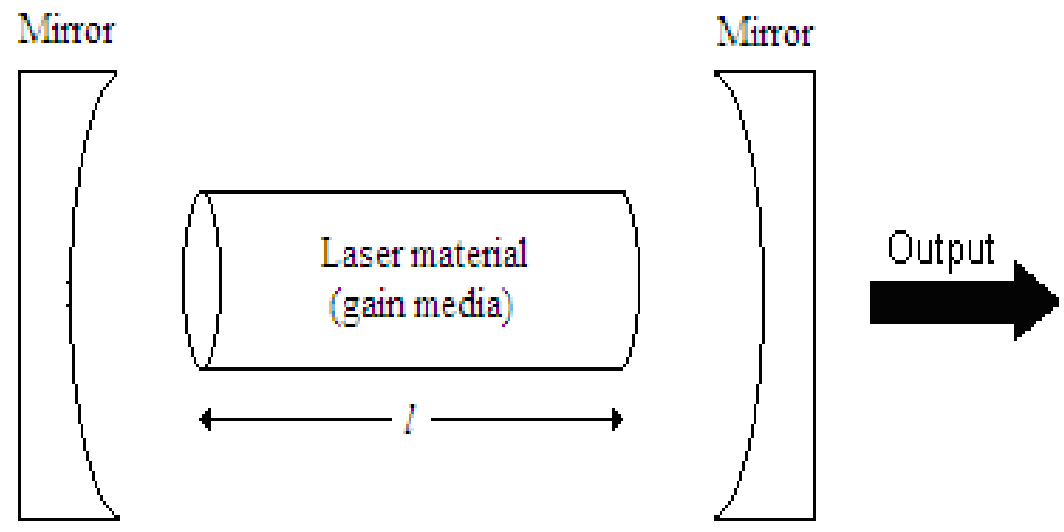

Figure-1 Laser oscillator

In laser oscillator, the pump inverts the electron population in the laser material, leading to energy storage in the upper laser level. If this energy is released to the optical beam by stimulated emission, amplification takes place. Having been triggered by some spontaneous radiation emitted along the axis of the laser, the system starts to oscillate if the feedback is sufficiently large to compensate for the internal and external losses of the system. The amount of feedback is determined by the reflectivity of the mirrors. Lowering the reflectivity of the mirror at the output end of the laser must be partially transparent for a fraction of the radiation to "leak out" or emerge from the oscillator. The role of the resonator is to maintain an electromagnetic field configuration whose losses are replenished by the amplifying medium through induced emission. Thus the resonator defines the spectral, directional, and spatial characteristics of the laser radiation, and the amplifying medium serves as the energy source. 


\section{OPERATION AT THRESHOLD}

A laser oscillator composed of two mirrors having a reflectivity $R_{1}$ and $R_{2}$ and an active material of length $l$, a gain per unit length $g(v)$ in the inverted laser material. In each passage through the material the intensity gains by a factor of $\exp (g l)$. At each reflection a fraction $1-R_{1}$ or $1-R_{2}$ of energy is lost. Starting at one point, the radiation will suffer two reflections before it can pass the same point in the original direction. The threshold condition is established by requiring that the photon density - after the radiation has traversed the laser material, been reflected by mirror with $R_{l}$, and returned through the material to be reflected by mirror with $R_{2}$, be equal to the initial photon density. Then on every complete two way passage of the light through the laser the loss will just equal the gain. The threshold condition is

$$
R_{l} R_{2} \exp (2 g l)=1
$$

The regenerative amplifier becomes unstable when the amplification per transit exceeds the losses. In this case oscillations will build up, starting from a small disturbance. Clearly, if the loop gain,

$$
G=R_{1} R_{2} \exp (2 g l)
$$

is larger than 1 , radiation of the proper frequency will build up rapidly until it becomes so large that the stimulated transition will deplete the upper level and reduce the value of $g$. The condition of steady state is reached if the gain per pass exactly balances the internal and external losses. This process is called gain saturation. In an oscillator a number of loss mechanisms are instrumental in attenuating the beam; the most important ones are reflection, scattering, and absorption losses in the mirrors, amplifying medium, and all other elements in the resonator, and diffraction losses [2].

It is convenient to lump all the non-output losses into a single parameter, the absorption coefficient per unit length $\alpha$. The condition for oscillation is then,

$$
R_{l} R_{2} \exp (g-\alpha) 2 l=1
$$

Here all the loss mechanisms are characteristized by a single parameter $\tau_{c}$, which is equal to the decay time constant of the radiation in a passive resonator. Resonators are characterized by quality factor $Q$, which is defined as the ratio of energy stored in the resonator to power dissipated from the resonator per unit angular frequency $\omega_{0}$. The resonator $Q$ defined in this way is equal to

$$
Q=2 \pi\left[1-\exp \left(\frac{-T_{0}}{\tau_{c}}\right)\right]^{-1} \approx \frac{2 \pi \tau_{c}}{T_{0}}=2 \pi v_{0} \tau_{c}
$$

Where $\omega_{0}=2 \pi v_{0}=2 \pi / T_{0}$. The loss mechanism, besides limiting the lifetime of the oscillation, causes a broadening of the resonance frequency. The width $\Delta v$ of the resonance curve at which the intensity has fallen off to half the maximum value is

$$
\Delta v=\left(2 \pi \tau_{c}\right)^{-1}
$$

Thus from equation (4) we obtain for the $Q$ value

$$
Q=\frac{v_{0}}{\Delta v}
$$

The decay time constant of the radiation $\tau_{c}$ can also be defined as the average lifetime of the photons in the resonator. A photon in the cavity will have some average lifetime in the cavity before being scattered or emitted or lost in other ways to the optical system. If we relate $\tau_{c}$ to the fractional power loss $\varepsilon$ per round trip, we obtain

$$
\varepsilon=\frac{{ }^{t} R}{\tau_{c}}
$$

Where $t_{R}=2 l^{\prime} / c$ is the round trip time of a photon in a resonator having an optical length $l^{\prime}$.Rearranging equation (5) yields

$$
2 g l=\ln \left(R_{l} R_{2}\right)+2 \alpha l(8)
$$

The expression on the right is the total fractional power loss per round trip. Since $2 g l=\varepsilon=t_{R} / \tau_{c}$, we obtain

$$
\tau_{c}=\frac{2 l^{\prime}}{c}\left[\ln \left(R_{1} R_{2}\right)^{-1}+2 \alpha l\right]^{-1}
$$

Miscellaneous losses, such as absorption and scattering at the mirrors and diffraction losses of the resonator can be thought of as leakage from the rear mirror. Hence the reduced reflectivity $R_{2}$ of the rear mirror, $R_{2}=1-L_{\mathrm{M}}$ takes into account the miscellaneous losses. In practice $L_{M}$ does not exceed a few percent. With the approximation

$$
\ln \left(1-L_{M}\right) \approx-L_{M}
$$

One can combine the optical losses in the cavity with the losses in the crystal: 
With this approximation (8) reduces to

$$
L=2 \alpha l+L_{M}
$$

$$
\tau_{c}=\frac{2 l^{\prime}}{c\left(L-\ln R_{1}\right)}
$$

From equations (9) and (10), the threshold condition (3) is expressed in the following form:

$$
2 g l=L-\ln R_{l} \approx T+L
$$

The approximation $-\ln R_{l} \approx T$ is valid only for values $R_{l}$ close to one.

\section{CIRCULATING POWER}

If the photons bounce back and forth between the mirrors for a long enough period, the laser will reach a steady-state condition and a relativity constant power will circulate between the mirrors. This circulating power is not absolutely constant. Part of it is lost when it hits the output mirror, and this lost power is replaced when the light passes through the gain medium. Figure- 2 shows how the circulating power varies inside the resonator. The circulating power drops at the output mirrors because part of the light is transmitted through the mirror. The remaining light travels through the gain medium where it is partially replenished. There is a small loss at the back mirror because no mirror is a perfect reflector. Then the light returns for a second pass through the gain medium, where it is fully restored to its previous level at point A.The power in the output beam is determined by the amount of circulating power and the transmission of the output mirror as:

$$
P_{\text {out }}=\tau P_{\text {circ }}
$$

Where $\tau$ the transmission of the output mirror and $P_{\text {out }}$ and $P_{\text {circu }}$ are the output and the circulating powers, respectively. According to Koechner [2], the output power is

$$
P_{\text {out }}=A I\left(1-R_{1}\right) R_{1}^{-\frac{1}{2}}
$$

This equation relates the output power obtained from the oscillator to the geometric mean power density Iinside the optical resonator. Here $A$ is the area of the front mirror.

From equation (13), the output power is proportional to the mirror transmission. But it does not mean that we can always increase the output power by increasing the mirror transmission, because as the transmission of the output mirror is increased, the circulating power will decrease. Whatever happens to the output power depends on whether the circulating power decreases faster than the mirror transmission increases, and this depends on the particular laser we are looking at. In fact, for any laser there will be an optimum value for the transmission of the output mirror that will produce the maximum possible output power [1].In Figure-2, the loop is closed, i.e., the circulating power is restored to precisely its initial value after a round trip through the laser resonator. This is true for any steady state, or continuous wave laser, where the circulating power can settle down to steady state behaviour. In most pulsed lasers the situation is different because energy moves so quickly from the population inversion to circulating power to output power that it never has time to reach equilibrium. If the circulating power is restored to its original value after a round trip of the resonator, the round-trip gain must be equal to the round-trip loss. If the round-trip gain is less than the round-trip loss, the laser will not lase. On the other hand, if the round-trip gain is greater than the round-trip loss, the gain will saturate until it is reduced to the same value as the round-trip loss.
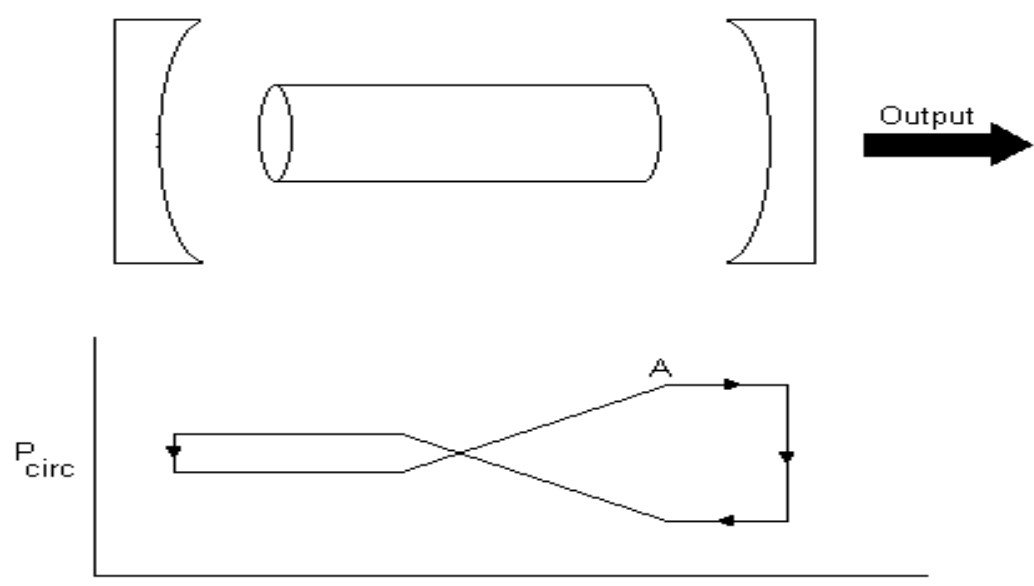

Figure-2 circulating power inside the resonator 


\section{RESONATORS MODES}

The light emitted by most lasers contains several discrete optical frequencies, separated from each other by frequency differences, which can be associated with different modes of the optical resonator. It is common practice to distinguish two types of resonator modes: "Longitudinal" modes differ from one another only in their oscillation frequency; and "Transverse" modes differ from one another not only in their oscillation frequency, but also in their field distribution in a plane perpendicular to the direction of propagation. Corresponding to a given transverse mode are a number of longitudinal modes which have the same field distribution as the given transverse mode but which differ in frequency.To describe the electromagnetic field variations inside optical resonators, the symbol $T E M_{m n q}$ or $T E M_{p l q}$ are used. The capital letters stands for "Transverse Electromagnetic Waves" and the first two indices identify a particular transverse mode, whereas $q$ describes a longitudinal mode. Because resonators that are used for typical lasers are long compared to the laser wavelength, they will, in general, have a large number of longitudinal modes. Therefore, the index $q$, which specifies the number of modes along the axis of the cavity, will be very high. The indices for the transverse modes, which specify the field variations in the plane normal to the axis, are very much lower and sometimes may be only the first few integers.The spectral characteristics of a laser, such as linewidth and coherence length, are primarily determined by the longitudinal modes; whereas beam divergence, beam diameter, and energy distribution are governed by the transverse modes. In general, lasers are multimode oscillators unless specific efforts are made to limit the number of oscillating modes. The reason for this lies in the fact that a very large number of longitudinal resonator modes fall within the bandwidth exhibited by the laser transition and a large number of transverse resonator modes can occupy the cross section of the active material [2].

\section{TRANSVERSE MODES}

The output spot of the laser beam is termed the transverse electromagnetic mode (TEM). Transverse modes are defined by the designation $T E M_{n m}$ for Cartesian coordinates. The integers $m$ and $n$ represent the number of nodes or zeros of intensity transverse to the beam axis in the vertical and horizontal directions. In cylindrical coordinates the modes are labelled $T E M_{p l}$ and are characterized by the number of radial nodes $p$ and angular nodes $l$. The higher the values of $m, n, p$, and $l$, the higher the mode orders. The lowest-order mode is the $T E M_{00}$ mode, which is a round mode with a Gaussian-like intensity profile in cross-section, with its maximum on the beam axis. However it is possible to operate on a wide variety of other transverse mode configurations. In these configurations, the output spot will have a much more peculiar shape[1].For mode with subscripts of 1 or more, intensity maxima occur that are off-axis in a symmetrical pattern. To determine the location and amplitudes of the peaks and nodes of the oscillation modes, it is necessary to employ higher-order equations, which either involve Hermit or Laguerre polynomials. The Hermit polynomials are used when working with rectangular coordinates, while Laguerre polynomials are more convenient when working with cylindrical coordinates. The transverse mode structure will be calculated using the paraxial approximation to the electromagnetic wave equations [3][2].

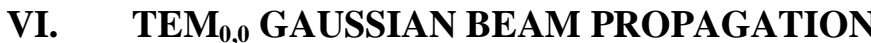

The lowest-order transverse mode is called the $T E M_{0,0}$ mode, the 00 -mode, the lowest-order mode, fundamental mode, or the Gaussian mode. This is the mode that is circular in transverse dimensions and has a Gaussian intensity profile. It is the mode that is most widely used in laser systems [4][5][6]. The decrease of the field amplitude with distance $r$ from the axis in a Gaussian beam is described by Koechner[2] as:

$$
\tilde{E}(r)=\tilde{E}_{0} \exp \left(\frac{-r^{2}}{w^{2}}\right)
$$

Thus, the distribution of power density is

$$
I(r)=I_{0} \exp \left(\frac{-r^{2}}{w^{2}}\right)
$$

The quantity $w$ is the radial distance at which the field amplitude drops to $1 / e$ of its value on the axis and the power density is decreased to $1 / e^{2}$ of its axial value. The parameter $w$ is often called the beam radius or "spotsize" and $2 w$, the beam diameter. According to Koechner [2]the fraction of the total power of a Gaussian beam which is contained in a radial aperture of $r=w, r=1.5 w$, and $r=2 w$ is $86.5 \%, 98.9 \%$ and $99.9 \%$. If a Gaussian beam is passed through a radial aperture of $3 w$, then only $10^{-6} \%$ of the beam power is lost due to the obstruction. Therefore a radial aperture in excess of three spot sizes is means an "infinite aperture". Although the intensity distribution is Gaussian in every propagating beam cross section, the width of the intensity profile changes along the axis. The Gaussian beam contracts to a minimum diameter $2 w_{0}$ at the beam waist where the phase front is planer. 


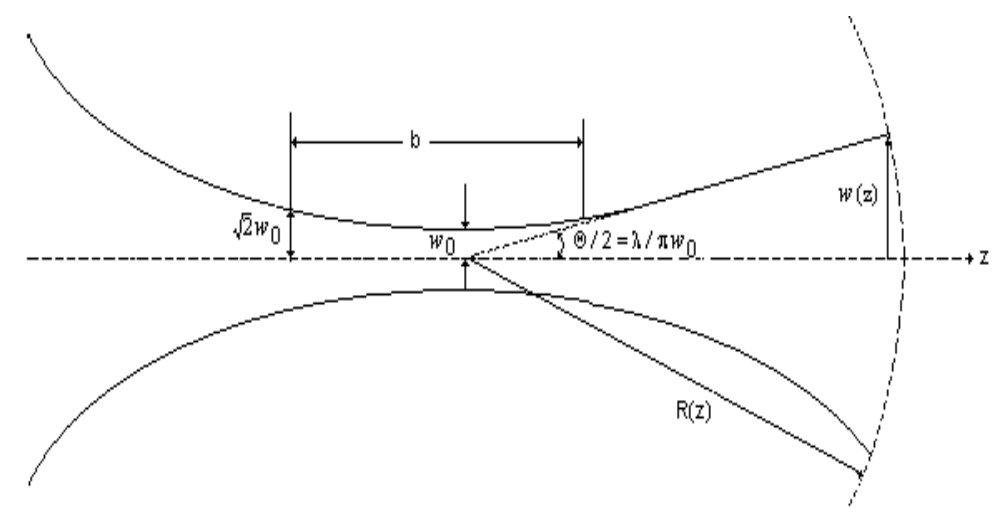

Figure-3 Gaussian beam

If one measures $z$ from this waist, the expansion laws for the beam assume a simple form. The spot size a distance $z$ from the beam waist expands as a hyperbola, which has the form

$$
w(z)=w_{0}\left[1+\left(\frac{\lambda z}{\pi w_{0}^{2}}\right)^{2}\right]^{1 / 2}
$$

Its asymptotes are inclined at an angle $\Theta / 2$ with the axis, as shown in Figure-3, and defines the far-field divergence angle of the emerging beam. The full divergence angle for the fundamental mode is given by

$$
\Theta=\lim _{z \rightarrow \infty} \frac{2 w(z)}{z}=\frac{2 \lambda}{\pi w_{0}}=1.27 \frac{\lambda}{\left(2 w_{0}\right)}
$$

From these considerations it follows that at large distances, the spot size increases linearly with $z$, and the beam diverges at a constant cone angle $\Theta$. The smaller the spot size $w_{0}$ at the beam waist, the greater the divergence. At sufficiently large distances from the beam waist the wave has a spherical wavefront appearing to emanate from a point on the beam axis at the waist. If $R(z)$ is the radius of curvature of the wavefront that intersects the axis at $z$, then

$$
R(z)=z\left[1+\left(\frac{\pi w_{0}^{2}}{\lambda z}\right)^{2}\right]
$$

In a Gaussian beam the wavefront has the same phase across its entire surface. According to Koechner[2], sometimes the properties of a $T E M_{00}$ mode beam are described by specifying a confocal parameter

$$
b=\frac{2 \pi w_{0}^{2}}{\lambda}
$$

Where $b$ is the distance between the points at each side of the beam waist for which $\quad w(z)=\sqrt{2} w_{0}$ (Figure-3). A laser operating at the $T E M_{00}$ mode will have a beam divergence according to Eq. (18). For a plane wavefront incident upon a circular aperture of diameter $D$, the full cone angle of the central (Airy) disc, defined at the first, minimum of the Fraunhofer diffraction pattern, is given by

$$
\Theta_{P}=\frac{2.44 \lambda}{D}
$$

the energy contained within this angle is about $84 \%$ of the total energy transmitted by the aperture.

Equations (18) and (21) are often confused, because various conventions have been adopted by different authors, with the equation

$$
\Theta_{R}=\frac{1.22 \lambda}{D}
$$

Which represents the half-cone angle of the Fraunhofer diffraction pattern, and also happens to be the "Rayleigh criterion" for the angular resolution of an optical instrument?

In laboratory work, a beam size is often obtained by measuring the diameter of the illuminated spot with a scale. This is not the spot size $2 w_{0}$ as defined by (17). There is no obvious visual cue to the magnitude of the spot size in the appearance of the illuminated spot. Thus, "spot size" and "size of the illuminated spot" are totally different concepts. The former is a property of the laser cavity; the latter is a subjective estimate. To 
measure the spot size, the illuminated spot is scanned with a photodetector behind a small pinhole. The resulting curve of intensity versus position of the pin-hole will yield a Gaussian curve from which the spot size $\omega_{0}$ be extracted by mathematical methods [6][7].

\section{RESONATOR CONFIGURATION}

The most commonly used laser resonators are composed of two spherical or flat mirrors facing each other. Let us consider the generation of the lowest-order mode by such a resonant structure. Once the parameters of the $T E M_{00}$ mode are known, all higher-order modes simply scale from it in a known manner. Diffraction effects due to the finite size of the mirrors are neglected.

The Gausssian beam shown in Figure-4, has a wavefront curvature of $R_{1}$ at a distance $t_{1}$ from the beam waist. If we put a mirror at $t_{1}$ whose radius of curvature equals that of the wavefront, then the mode shape has not been altered. To proceed further, we can go along the $z$ axis to another point $t_{2}$ where the $T E M_{0 o}$ mode has a radius of curvature $R_{2}$, and place there a mirror whose radius of curvature $R_{2}$ equals that of the spherical wavefront at $t_{2}$. Again the mode shape remains unaltered.

Therefore, to make a resonator, we simply insert two reflectors, which match two of the spherical surface defined by (19). Alternately, given two mirrors separated by a distance $L$, if the position of the plane $z=$ 0 and the value of the parameter $w_{0}$ can be adjusted so that the mirror curvatures coincide with the wavefront surfaces, we will have found the resonator mode.Kogelnik and $\mathrm{Li}$ [7] gave formulas, which relate the mode parameters $w_{1}, w_{2}, w_{0}, t_{1}$ and $t_{2}$ to the resonator parameters $R_{1}, R_{2}$ and $L$. as illustrated in Figure- $4, w_{1}$ and $w_{2}$ are the spot radii at mirrors $M_{1}$ and $M_{2}$, respectively; $t_{1}$ and $t_{2}$ are the distances of the beam waist described by $w_{0}$ from mirrors $M_{1}$ and $M_{2}$ which are separated a distance $L$. Labeling conventions are that concave curvatures are positive. The beam radii at the mirrors are given by

$$
\left.\begin{array}{l}
w_{1}^{4}=\left(\frac{\lambda R_{1}}{\pi}\right)^{2} \frac{R_{2}-L}{R_{1}-L}\left(\frac{L}{R_{1}+R_{2}-L}\right) \\
w_{2}^{4}=\left(\frac{\lambda R_{2}}{\pi}\right)^{2} \frac{R_{1}-L}{R_{2}-L}\left(\frac{L}{R_{1}+R_{2}-L}\right)
\end{array}\right\}
$$

The radius of the beam waist, which is formed either inside or outside the resonator, is given by

$$
w_{0}^{4}=\left(\frac{\lambda}{\pi}\right)^{2} \frac{L\left(R_{1}-L\right)\left(R_{2}-L\right)\left(R_{1}+R_{2}-L\right)}{\left(R_{1}+R_{2}-2 L\right)^{2}}
$$

The distances $t_{1}$ and $t_{2}$, between the waist and the mirrors, measured positive as shown in Figure- 4 , are

$$
t_{1}=\frac{L\left(R_{2}-L\right)}{\left(R_{1}+R_{2}-2 L\right)}, t_{2}=\frac{L\left(R_{1}-L\right)}{\left(R_{1}+R_{2}-2 L\right)}
$$

These equations treat the most general case of a resonator. These are many optical resonator configurations for which (23) - (25) are greatly simplified. Figure-5 shows some of the most commonly used geometry of resonators [8].

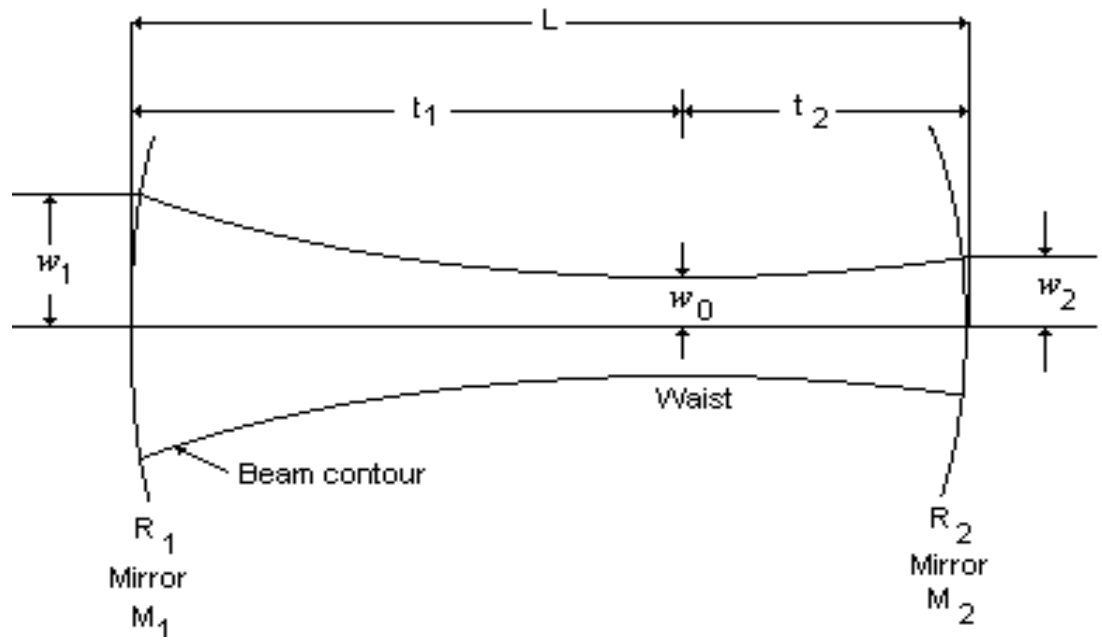

Figure-5 Resonator with mirrors of unequal curvature 


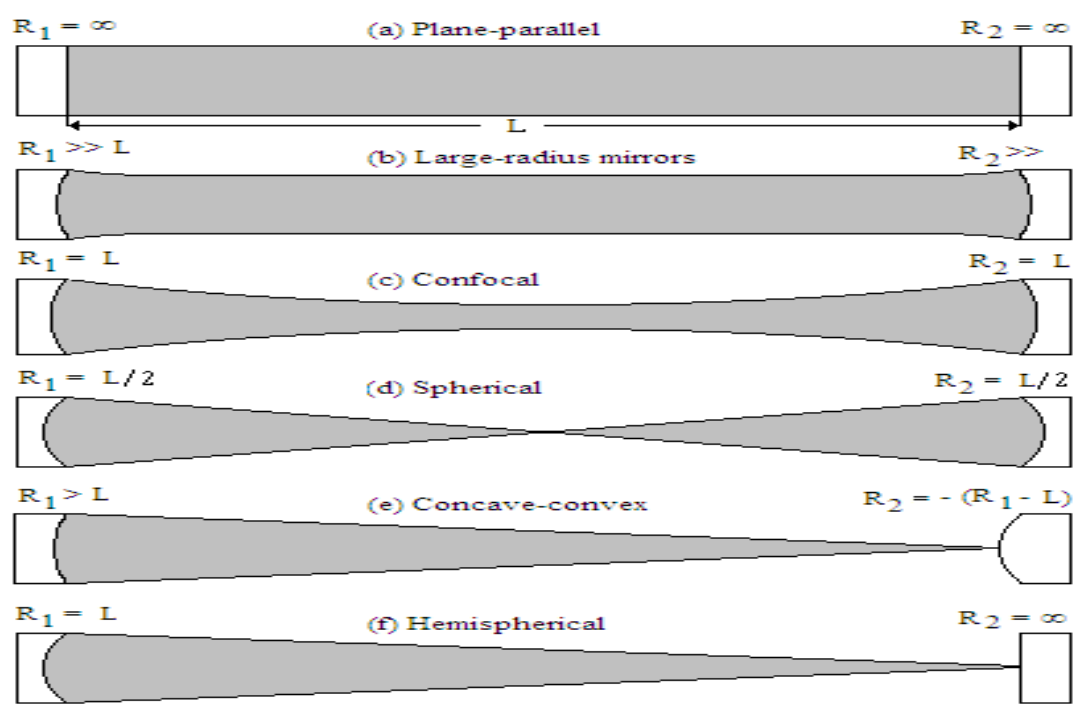

Figure-6 Resonator configurations giving uniphase wavefronts

VIII. STABILITY OF LASER RESONATORS

A stable resonator is, by definition, one in which a ray is trapped between the mirrors by their curvature. The word stable implies nothing about a resonator's sensitivity to misalignment nor about an absence of fluctuations in its output power. Most lasers have stable resonators, in which the curvatures of the mirrors keep the light concentrated near the axis of the resonator. If the path of the light ray is traced between the mirrors of a stable resonator, it is found that the ray is eventually reflected back toward the resonator axis by the mirrors as shown in Figure-7. The only way light can escape from the resonator is to go through one of the mirrors.

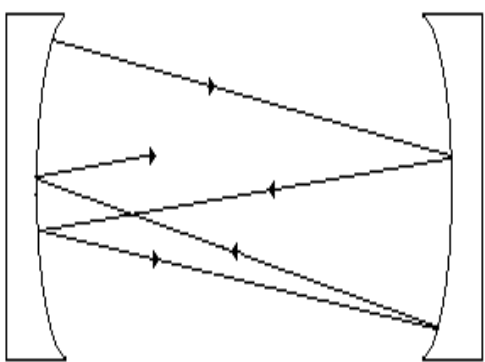

Stable Resonator

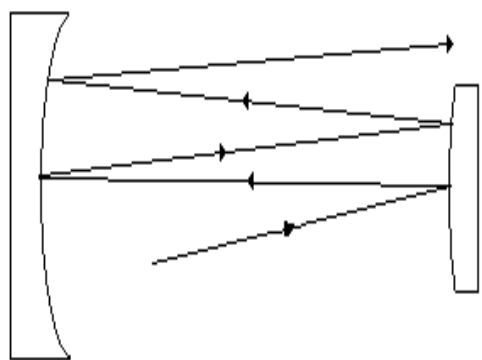

Unstable Resonator

Figure-7 Stable \& Unstable Resonator

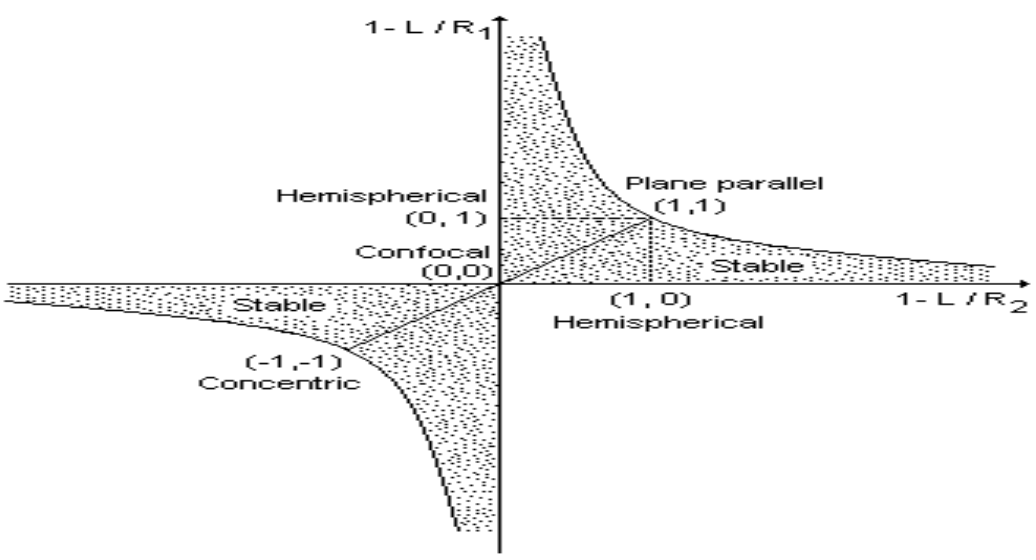

Figure-8 Stability diagram for the passive laser resonator 


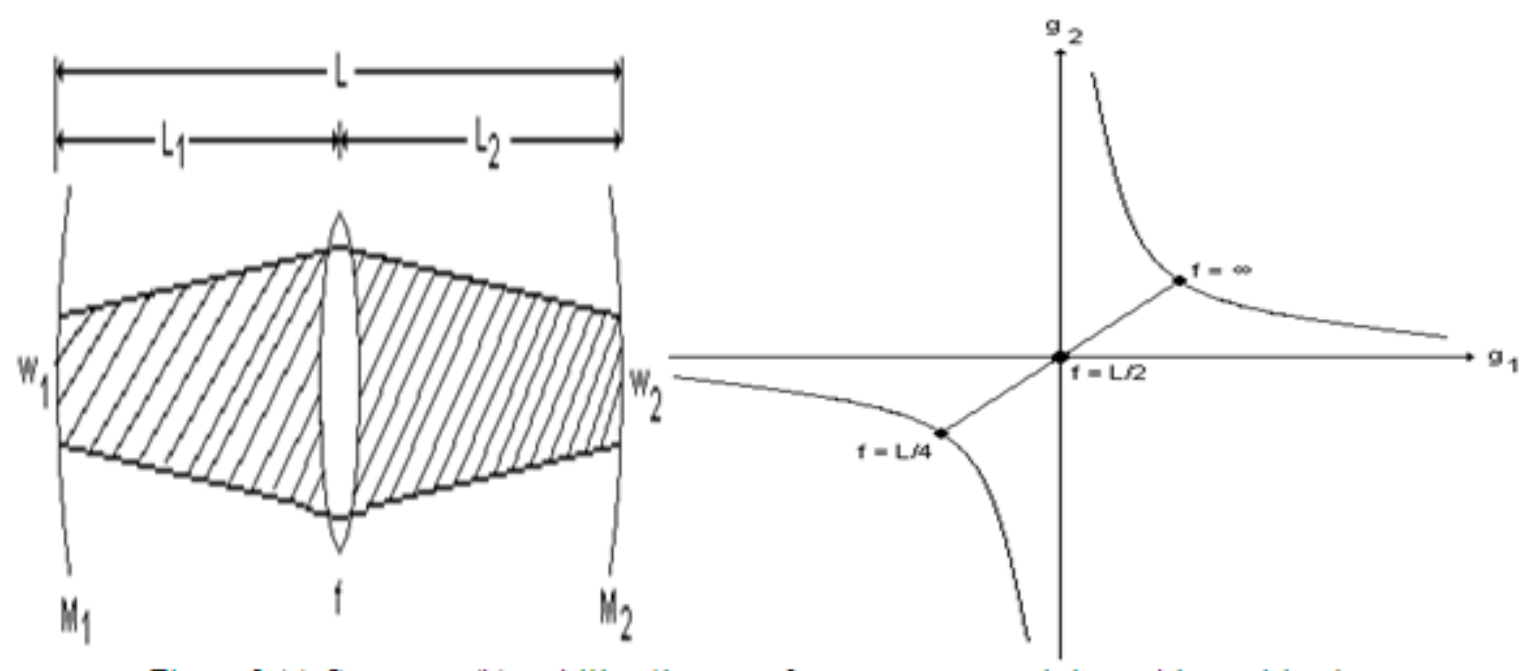

Figure-9 (a) Geometry (b) stability diagram of a resonator containing a thin positive lens

In the unstable resonator, the light rays keep on moving away from the resonator axis until eventually they miss the small convex mirror altogether. The output beam from this resonator will have a doughnut-like shape with a hole in the middle caused by the shadow of the small mirror. Thus unstable resonators are designed in a ways that avoid the hole-in-the-center beam. The advantage of unstable resonators is that they usually produce a larger beam volume inside the gain medium so the beam can interact with more of the population inversion and thereby produce more output power. Unstable resonators are usually used only with high-power, pulsed gas and solid-state lasers [1]. Light rays that bounce back and forth between the spherical mirrors of a laser resonator experience a periodic focusing action. The effect on the rays is the same as in a periodicsequence of lenses. Rays passing through a stable sequence of lenses are periodically refocused. For unstable systems the rays become more and more dispersed the further they pass through the sequence. In an optical resonator operated in the stable region, the waves propagate between reflectors without spreading appreciably. This fact can be expressed by a stability criterion [2][3]

$$
0<\left(1-\frac{L}{R_{1}}\right)\left(1-\frac{L}{R_{2}}\right)<1
$$

To show graphically which type of resonator is stable and which is unstable, it is useful to plot a stability diagram on which each particular resonator geometry is represented by a point. This is shown in Figure-7, where below parameters are drawn as the coordinate axes.

$$
g_{1}=1-\frac{L}{R_{1}} ; \quad g_{2}=1-\frac{L}{R_{2}} ;
$$

All cavity configurations are unstable unless they correspond to points in the area enclosed by a branch of the hyperbola $g_{1} g_{2}=1$ and the coordinate axes. The origin of the diagram represents the confocal system. The low-loss regions of Figure-8 represent the following three sets of possible mirror geometries:

1. Both mirrors are concave, and the center of curvature of each mirror lies beyond the other mirror.

2. Both mirrors are concave, and the center of curvature of each mirror lies between the other mirror and the center of curvature of the other mirror.

3. One mirror is convex and one is concave, and the center of curvature of the concave mirror lies between the convex mirror and the center of curvature of the convex mirror. It turns out that the mirror configurations which satisfy the stability criterion are those for which the wavefront and mirror curvature are matched.

\section{ACTIVE RESONATOR}

Introducing an active element into the resonator, such as a laser crystal, in addition to altering the optical length of the cavity, will perturb the mode configuration, since the active material possesses a saturable, nonuniform gain and exhibits thermal lensing and birefringence. In high-gain, giant-pulse lasers, gain saturation at the center of a $T E M_{00}$ mode can lead to a flattening of the intensity profile [9]. Also, pump nonuniformities leading to a nonuniform gain distribution across the beam will lead to non-Gausssian output intensity profiles. Theoretical and experimental investigations have shown that in solid-state lasers the governing mechanisms which distort the mode structure in the resonator are the thermal effects of the laser rod. Optical pumping leads to a radial temperature gradient in the laser rod. As a result, in $c w$ and high average power systems, the rod is 
acting like a positive thick lens of an effective focal length $f$, which is inversely proportional to the pump power [10].

The theory necessary to analyze resonators that contain optical elements other than the end mirrors has been developed by Kogelnik [11]. This theory is applied to the case of a resonator containing an internal thin lens. To a first approximation, this lens can be thought of as representing the thermal lensing introduced by the laser rod. The more complex case of a distributed thick lens which more adequately describes thermal lensing has been treated in [12].Beam properties of resonators containing internal optical elements are described in terms of an equivalent resonator composed of only two mirrors. The pertinent parameters of a resonator equivalent to none with an internal thin lens are

$$
g_{1}=1-\frac{L_{2}}{f}-\frac{L_{0}}{R_{1}} ; g_{2}=1-\frac{L_{1}}{f}-\frac{L_{0}}{R_{2}}
$$

where $L_{0}=L_{1}+L_{2}-\left(L_{1} L_{2} / f\right)$ and $f$ is the focal length of the internal lens; $L_{1}$ and $L_{2}$ are the spacing between mirrors $M_{1}, M_{2}$ and the lens as shown in Figure-9a. In any resonator, the $T E M_{00}$ mode spot size at one mirror can be expressed as a function of the resonator parameters

$$
w_{1}^{2}=\frac{\lambda L}{\pi}\left[\frac{g_{2}}{g_{1}\left(1-g_{1} g_{2}\right)}\right]^{1 / 2}
$$

the ratio of the spot sizes at the two mirrors is

$$
\frac{w_{1}^{2}}{w_{2}^{2}}=\frac{g_{2}}{g_{1}}
$$

the stability condition (26) remains unchanged. As an example we will consider a resonator with flat mirrors $\left(R_{1}\right.$ $\left.=R_{2}=\infty\right)$ and a thin lens in the center $\left(L_{1}=L_{2}=L / 2\right)$. From (28) we obtain

$$
\begin{gathered}
g=g_{1}=g_{2}=1-\frac{L}{2 f}, \\
w_{1}^{2}=w_{2}^{2}=\left(\frac{\lambda L}{\pi}\right)\left(1-g^{2}\right)^{-1 / 2}
\end{gathered}
$$

For $f=\infty$ the resonator configuration is plane-parallel; for $f=L / 2$ we obtain the equivalent of a confocal resonator; and $f=L / 4$ the resonator corresponds to a spherical configuration.

The mode size in the resonator will grow to infinity as the mirror separation approaches four times the focal length of the laser rod. Figure-9b shows the location of a plane-parallel resonator with an internal lens of variable focal length in the stability diagram. Above types of resonators are restricted to devices having axial symmetry with respect to the beam axis. Resonators that contain inclined surfaces, such as Brewster-ended rods, polarizers, prisms, etc., lack axial symmetry. The effect of these asymmetric devices is to produce astigmatic beams, i.e., beams that have different spot sizes, wavefront curvatures, and beam waist positions in two orthogonal directions. Axially asymmetric laser cavities have been analyzed by Hanna and Kogelnik [13][14].

\section{DIFFRACTION LOSSES}

In any real laser resonator some part of the laser beam will be lost either by spillover at the mirrors or by limiting apertures, such as the lateral boundaries of the active material. These losses will depend on the diameter of the laser beam in the plane of the aperture and the aperture radius. If we take a finite aperture of radius $a$ within the resonator into account, the diffraction losses depend on four parameters $R_{1}, R_{2}, L$ and $a$, which describe the resonator; and on three parameters $\lambda, m$ and $n$, characterizing the particular optical beam present in the resonator. Fortunately, the losses depend only on certain combinations of these parameters. These combinations are the so-called Fresnel number [2]

$$
N=\frac{a^{2}}{\lambda L}
$$

And the quantities which were defined in (27). The parameter $N$ can be thought of as the ratio of the acceptance angle $(a / L)$ of one mirror as viewed from the center of the opposing mirror to the diffraction angle ( $\lambda / a)$ of the beam. Therefore, when $N$ is small, especially if $N<1$, the loss factor will be high because only a portion of the beam will be intercepted by the mirrors. When $N$ is large, the losses will be low for the stable resonator configurations and large for the unstable resonators. If two resonators have the same values of $N, g_{1}$ and $g_{2}$, then they have the same diffraction loss, the same resonant frequency, and the same mode patterns. The fractional energy loss per transit due to diffraction effects for the lowest-order mode of a resonator with identical 
mirrors $\left(g_{1}=g_{2}=g\right)$ is given by Li [15]. For the plane-parallel resonator with circular aperture, an analytical expression of the diffraction losses for large Fresnel numbers $(F \geq 1)$ has been derived by Vainshtein [16]:

$$
\alpha=8 k_{p l}^{2} \frac{\delta(M+\delta)}{\left[(M+\delta)^{2}+\delta^{2}\right]^{2}}
$$

Where $\delta=0.824, M=(8 \pi N)^{1 / 2}$ and $k_{p l}$ is the $(p+l)$ th zero of the Bessel function of order $l$. The diffraction losses in a confocal resonator for a number of low-order modes are shown by McCumber [17]. He showed that all have very large losses for small $N$ and that the losses drop with increasing $N$. However, the losses for the higher-order modes drop less rapidly than the losses for the lower-order modes.

\section{RESONATOR SENSITIVITY}

There are two contexts in which the term "stability" is used. First, laser resonators are said to be optically stable or unstable depending on the values of $g_{1}, g_{2}$. Second the mode size and position are sensitive to mechanical and optical perturbations of the optical elements. When designing resonators for an optimum mode size, it will be of the utmost importance to consider the resonator sensitivity to these mechanical and optical perturbations. Usually one is interested in the sensitivity of the resonator to two common types of perturbations: first, a time-varying thermal lensing effect caused by the laser rod, and second, misalignments of the resonator mirrors. The former perturbation leads mainly to a change in mode size and beam divergence [12][18], whereas the latter perturbation leads to a lateral displacement and angular tilt of the output beam [19] which causes an increase of the diffraction losses and, therefore, a reduction of output power. First-order effects on the modes as a function of cavity perturbation are usually obtained by evaluation of sensitivity matrix.

Considering first the resonator's sensitivity to lensing effects, a resonator is insensitive to axial perturbations if the spot size $w_{1}$ is insensitive to changes of $g_{1}$ and $g_{2}$ [eqs. (29), (31)]. A calculation of the relative sensitivities of various resonators to small changes in mirror radii of curvature has been carried out by Chesler and Mayden [18] and Steffen et al.[12]. A perturbation within the resonator producing changes in mirror radii is equivalent to the introduction of a lens of some focal length $f$. In order for the resonator to have a low sensitivity to axial perturbations, i.e., small spot size changes for large changes of $g_{1}$ and $g_{2}$, it is necessary that $d w_{l} / d f=0$. This condition is met for resonator geometries, which satisfy the following equation:

$$
2 g_{2} g_{1}-1+\left(\frac{g_{1}}{g_{2}}\right)\left(\frac{L_{1}}{L_{2}}\right)^{2}+\frac{2 g_{1} L_{1}}{L_{2}}=0
$$

One particular resonator satisfying (34) is determined by

$$
g_{1} g_{2}=\frac{1}{2} ; \quad L_{1}=0
$$

In this case the internal lens is either absent or located at the surface of mirror $R_{1}$. Steffen et al. [12] shows that the curves, which are obtained from (29), reveal that the spot size is fairly insensitive to variations of $g_{1}$ and $g_{2}$ for resonator configurations which can be represented by points on the hyperbola $g_{1} g_{2}=0.5$. Large spot sizes $w_{1}$ are obtained for resonators with large $g_{2}$ values. From (27) follows that in order for $g_{2}>1$, the radius of curvature of mirror $R_{2}$ has to become negative, which indicates a convex mirror according to our labelling convention.

The resonator sensitivity to mirror misalignment is related to the fact that the mode axis must be normal to each of the two mirrors. This can be satisfied only if the ray is incident along a line, which passes through the center of curvature of the mirrors. Considering a typical resonator of mirror radii $R_{1}$ and $R_{2}$ and separation $L$, as shown in Figure-10, a rotation of mirror $M_{1}$ through an angle $\Theta$ rotates the line joining the centers of curvatures of the two mirrors through an angle $\phi$ and causes a linear displacement $x$ and $y$. Small-angle approximations are valid, and we have

$$
x=\frac{R_{1}\left(R_{2}-L\right) \Theta}{R_{1}+R_{2}-L}, \quad y=\frac{R_{1} R_{2} \Theta}{R_{1}+R_{2}-L}
$$

For a resonator with large radius mirrors of equal radii $R_{1}=R_{2}=R$ we obtain,

$$
x=y=\frac{R \Theta}{2}
$$

If one of the mirrors is slightly tilted, the entire mode is displaced parallel to the resonator axis. For a confocal resonator $\left(R_{1}=R_{2}=L\right)$ we have

$$
x=0 \quad \text { and } \quad y=L \Theta
$$




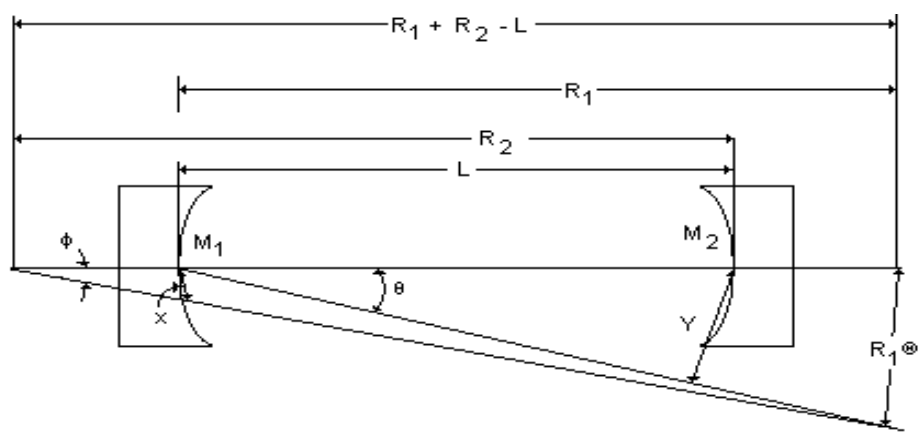

Figure-10 Mirror alignment parameters

In this case the mirror being tilted represents the pivot point for the mode axis. If the flat mirror of a hemispherical resonator $\left(R_{1} \approx \infty, R_{2} \approx \mathrm{L}\right)$ is tilted, we obtain

$$
x=\left(R_{2}-L\right) \Theta \approx 0, \quad y=L \Theta
$$

Comparing the sensitivity to the angular tilt of the various resonator configurations, it is found that, for example, a large-radius mirror resonator with $R=10 \mathrm{~L}$ is five times more sensitive to tilt than the confocal and hemispherical resonators.

Table-1 Performance of a cw Nd:YAG at different transverse modes

\begin{tabular}{|l|c|c|c|c|c|c|}
\hline & $\mathrm{TEM}_{00}$ & $\mathrm{TEM}_{01^{*}}$ & $\mathrm{TEM}_{10}$ & $\mathrm{TEM}_{11^{*}}$ & $\mathrm{TEM}_{20}$ & $\mathrm{TEM}_{21^{*}}$ \\
\hline Aperture size [mm] & 1.4 & 1.6 & 1.8 & 2.0 & 2.2 & 2.3 \\
\hline Laser output power [W] & 1.5 & 2.4 & 3.5 & 4.5 & 5.5 & 6.0 \\
\hline Beam divergence [mrad] & 1.9 & 2.3 & 2.8 & 3.1 & 3.4 & 3.6 \\
\hline Brightness [MW/cm $\left.{ }^{2} \mathrm{sr}\right]$ & 28 & 18 & 20 & 18 & 15 & 15 \\
\hline
\end{tabular}
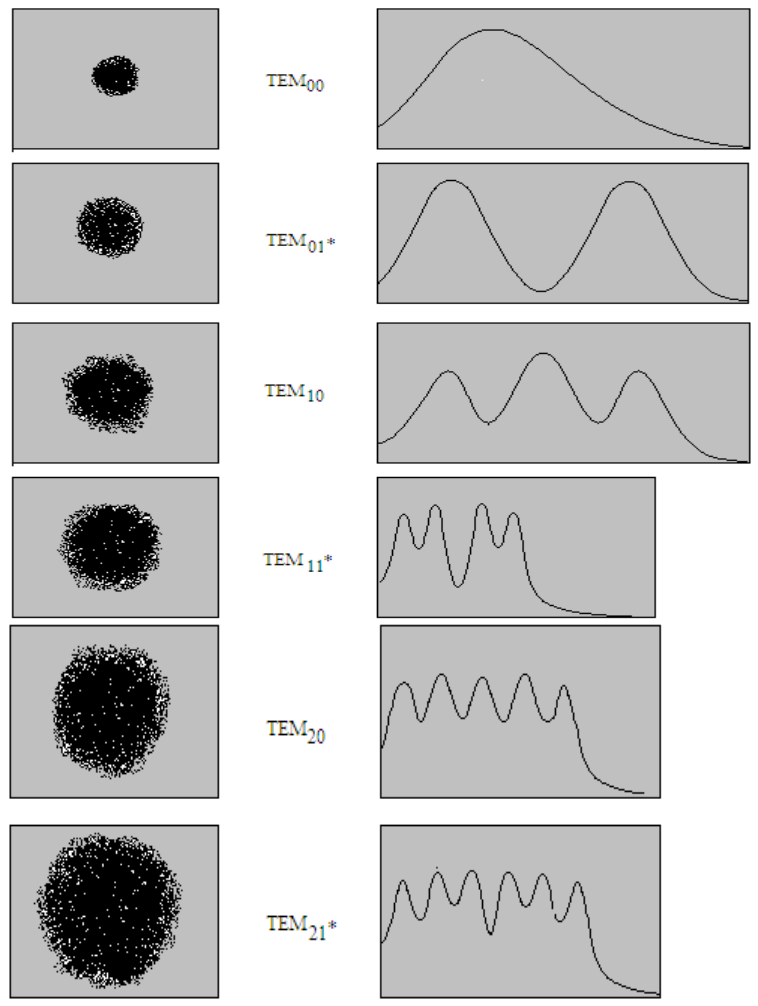

Figure-11 Mode patterns and beam intensity distribution of a cw Nd: YAG 
Freiberg et al.[19] shows that, the alignment tolerances of a resonator with relatively short-radius mirrors. Furthermore, a plano-concave resonator is more sensitive to misalignment than a resonator with to curved mirrors. Also, alignment tolerance becomes progressively less stringent for higher-order modes. Bleousova and Danilov [18] shows that, the higher-order mode is less sensitive to mirror misalignment and, furthermore, that the confocal resonator has the highest tolerance in terms of misalignment. Again, the confocal resonator is far more forgiving for mirror misalignment than the other types. Therefore the drop in output power of a pulsed Nd:YAG laser as a function of mirror misalignment for different mirror combination. As is to be expected, the resonator containing two curved mirrors is least sensitive to misalignment.Experiments have shown that reduction of the angular divergence of the beam is a means of transverse-mode control. Mode selectivity can be obtained by the sharp angular variations of the internal reflectivity near the critical angle of prisms or Lummer-Gehrke plate [20].It is important to have a perfectly aligned system with clean and damagefree optical surfaces. Slight misalignments, tilts, or imperfections (dust particles) of laser reflectors can cause changes in the mode character of the output, favoring higher-orders. For example, a particle or a damage spot located at the center of the beam can prevent oscillation in the $\mathrm{TEM}_{00}$ mode and cause oscillation at the TEM $M_{01}$ * mode [21].

To illustrated the effect of mode selection on the performance of a laser system, Koechner [2] shows in Figure-11, the mode pattern and radial intensity distributions from a $c w$-pumped Nd:YAG laser. Shown are the first six modes from the laser, which were obtained by successively increasing the mode-selecting aperture. The mode patterns were taken in the far field ( $20 \mathrm{~m}$ away from the laser) by photographing the light striking a ground-glass surface through an infrared image intensifier. The radial intensity distribution of the transverse mode patterns was observed by sweeping the laser beam across a small-aperture detector using the rotating plane mirror and displaying the detector signal on an oscilloscope. The system delivered a maximum of $8.5 \mathrm{~W}$ of multimode power. Mode selection was accomplished by insertion of different-size apertures into the cavity. The various aperture sizes and the corresponding output powers, beam divergences, and mode structures are summarized in Table-1.

\section{CONCLUSION}

When mirrors are placed at the ends of an amplifying medium, they not only effectively increase the length of the gain medium but also place boundary conditions upon the electromagnetic field (the laser beam) that develops between the two mirrors. These boundary conditions are similar as for the modes that develop within a cavity in radiative thermal equilibrium; similar modes also develop within the laser cavity. The conditions are such that the electric field must be zero at the reflecting surfaces of the mirrors. Therefore we begin the analysis by considering what happens when a beam of light is incident upon a two-mirrored cavity, known as a Fabry-Perot resonator, with no optical elements between the mirrors. We will then consider the effect of placing an amplifying medium between the mirrors.

\section{REFERENCES}

[1]. C. Break Hitz, Understanding Laser Technology, (PennWell Publishing Company, Tulsa, Oklahoma).

[2]. Walter Koechner, Solid State Laser Engineering, ${ }^{\text {rh }}$ ed., (Springer Berlin 1999).

[3]. K. J. Kuhn; Laser Engineering, Prentice Hall Pub., 1998.

[4]. Amnon Yariv, Optical Electronics, $\mathbf{4}^{\text {th }}$ ed. (Philadelphia, PA; Saunders College Publishing, 1991).

[5]. Joseph T. Verdeyen; Laser electronics, $\mathbf{2}^{\text {nd }}$ ed. (Englewood Cliffs, NJ; Printice-Hall, 1989).

[6]. H. Kogelnik and T. Li; Proc. IEEE,54, 1312 (1966).

[7]. J. S. Kruger; Electro-Opt. Syst.Designs12, (September 1972).

[8]. J. R. Leger, D. Chen, and K. Dai, Opt. Lett. 19, 1976 (1994).

[9]. A. G. Fox, T. Li; IEEE J. Quant. Electr. QE-2, 774 (1966).

[10]. P. A. Belanger, R. L. Lachance and C. Pare, Opt. Lett. 17, 739 (1992).

[11]. H. Kogelnik; Bell Syst. Tech. J. 44, 455 (1965).

[12]. J. Steffen, J. P. Lortscher, G. Herziger; IEEE J. Quant. Electr. QE-8, 239 (1972).

[13]. D. C. Hanna; IEEE J. Quant. Electr. QE-5, 483 (1969).

[14]. H. W. Kogelnik; IEEE J. Quant. Electr. QE-8, 373 (1972).

[15]. T. Li.; Bell Syst. Tech. J. 44, 917, (1965).

[16]. L. A. Vainshtein; JETP 44, 1050 (1963); JETP 17, 709 (1963).

[17]. D. E. McCumber; Bell Syst. Tech. J. 44, 333 (1965).

[18]. R. B. Chesler, D. Maydan; J. Appl. Phys. 43, 2254 (1972).

[19]. R. J. Freiberg, A. S. Halsted; Appl. Opt. 8, 355 (1969).

[20]. L. G. DeShazer, E. A. Maunders; Appl. Opt. 6, 431 (1967).

[21]. H. K. V. Lotsch; Japan J. Appl. Phys, 4, 435 (1965). 